\title{
Fatores socioeconômicos, demográficos, nutricionais e de atividade física no controle glicêmico de adolescentes portadores de diabetes melito tipo 1
}

\author{
Socioeconomic, demographic, nutritional, and physical activity factors \\ in the glycemic control of adolescents with type 1 diabetes mellitus
}

Rosana de Morais Borges Marques', Nélida Schmid Fornés', Maria Luiza Ferreira Stringhini ${ }^{1}$

1 Faculdade de Nutrição, Universidade Federal de Goiás (UFG), Goiânia, GO, Brasil

\author{
Correspondência para: \\ Rosana de Morais Borges Marques \\ Rua 1, 188, ap. 503, Setor Oeste, \\ 74115-040 - Goiânia, GO, Brasil \\ rosanambm@gmail.com \\ Recebido em 20/Set/2010 \\ Aceito em 21/Fev/2011
}

\begin{abstract}
RESUMO
Objetivo: Identificar a associação de condições socioeconômicas, demográficas, nutricionais e de atividade física sobre o controle glicêmico de adolescentes portadores de DM1. Sujeitos e métodos: Estudo transversal com 71 adolescentes com diagnóstico clínico de diabetes tipo 1. Foram obtidos dados socioeconômicos, demográficos e antropométricos. 0 controle glicêmico foi classificado pelo índice de hemoglobina glicada (A1C). Foram aplicados quatro recordatórios de 24 horas de consumo alimentar e de atividade física. Resultados: A A1C foi inadequada para a maioria dos adolescentes. A menor escolaridade do cuidador influenciou para o controle glicêmico inadequado. Pacientes com menor dose de insulina apresentaram melhor controle glicêmico. A alimentação observada foi hipoglicídica e hiperlipídica. A maioria dos pacientes era sedentária. Conclusão: Fatores relacionados à educação, à insulina e à alimentação influenciaram no controle glicêmico. Arq Bras Endocrinol Metab. 2011;55(3):194-202
\end{abstract}

Descritores

Adolescente; diabetes melito tipo 1; estado nutricional; fatores socioeconômicos; glicemia

\begin{abstract}
Objective: To identify the association of socioeconomic, demographic, nutritional and of physical activity factors in the glycemic control of adolescents with T1DM. Subjects and methods: Sectional study of 71 adolescents with type 1 diabetes. Socioeconomic, demographic and anthropometric data were obtained. The glycemic control was classified by the index of glycated hemoglobin (A1C). Four 24-hours recalls of food consumption and physical activity were applied. Results: The $\mathrm{A} 1 \mathrm{C}$ was inadequate for the majority of the adolescents. The low educational level of the caregivers influenced the inadequate glycemic control. Patients with lower insulin dose presented better glycemic control. The food consumption was high of fat and poor of carbohydrate. Most of the patients were sedentary. Conclusion: Factors related to education, insulin and food consumption influenced the glycemic control. Arq Bras Endocrinol Metab. 2011;55(3):194-202

Keywords

Adolescent; diabetes mellitus, type 1; nutritional status; socioeconomic factors; blood glucose
\end{abstract}

\section{INTRODUÇÃO}

mortalidade por doenças não transmissíveis vem auAmentando no Brasil e, dentre as causas de morte, o diabetes melito (DM) está entre as dez principais (1).
Estima-se que no Brasil existam aproximadamente $8 \mathrm{mi}$ lhões de portadores de diabetes, e que $5 \%$ a $10 \%$ desses são acometidos pelo diabetes tipo 1 (DMl) (2). Esse tipo de diabetes é caracterizado pela redução total ou 
parcial na produção de insulina e é o distúrbio endocrinometabólico crônico mais frequente na infância (3).

$\mathrm{O}$ diagnóstico de $\mathrm{DMl}$ ocorre principalmente na adolescência, mas sua incidência tem aumentado na infância. Com a manifestação clínica cada vez mais precoce, a perda da qualidade de vida desses jovens e o risco de morbidade e mortalidade são maiores, em decorrência à exposição prolongada a elevados níveis glicêmicos (4).

Burnet e cols. (5) ressaltaram que o maior risco de morte encontrado para pacientes diagnosticados na adolescência pode refletir um pobre controle metabólico e pouca participação dos pais no manejo da doença. Portanto, o adequado controle metabólico no DMl é fundamental para prevenir a ocorrência de episódios agudos de hiperglicemia ou hipoglicemia, como também para impedir ou retardar o desenvolvimento de doenças como neuropatias, nefropatias, obesidade, dislipidemia e doenças cardiovasculares.

Considerando a importância do tratamento de jovens portadores de DMl para promover condições saudáveis de desenvolvimento, crescimento e prevenção de comorbidades, o conhecimento de fatores ambientais e biológicos dessa população é imprescindível para determinação da melhor conduta terapêutica. Este trabalho teve como objetivo identificar a associação de condições socioeconômicas, demográficas, nutricionais e de atividade física sobre o controle glicêmico de adolescentes portadores de DMl.

\section{MÉTODOS}

\section{Delineamento e população}

Estudo observacional descritivo com 84 adolescentes, por amostra de conveniência, atendidos nos ambulatórios de endocrinologia do Hospital das Clínicas (HC) da Universidade Federal de Goiás (UFG) e do Hospital Geral de Goiânia (HGG), em Goiânia, Goiás, durante o período de abril de 2008 a julho de 2009.

Este estudo fez parte de um projeto intitulado "Validade e reprodutibilidade de um questionário quantitativo de frequência alimentar para adolescentes portadores de diabetes tipo I", em que tanto os pacientes do HC quanto do HGG apresentavam semelhanças quanto a idade, escolaridade, renda e índice de massa corporal.

Seguindo os critérios de inclusão de idade e diagnóstico clínico de diabetes tipo 1 (DMl), foram con- siderados aptos para participar do estudo adolescentes com idade entre 10 e 19 anos, de ambos os gêneros. Foram excluídos aqueles com diagnóstico de outros tipos de diabetes, doença celíaca, déficit de hormônio do crescimento, alterações cromossômicas e gestantes.

\section{Avaliação socioeconômica e demográfica}

Os dados socioeconômicos e demográficos foram obtidos por meio de entrevista direta com os adolescentes e responsáveis, na qual foi aplicado formulário próprio desenvolvido para esse fim. As perguntas se referiam a idade, escolaridade (do paciente e responsável), renda per capita mensal, Classificação Econômica Brasileira (CEB) (6) e condições de moradia.

\section{Avaliação antropométrica}

As medidas antropométricas foram realizadas pelo mesmo pesquisador, em duplicata, não consecutivas, e calculadas as médias, exceto para a dobra cutânea tricipital (DCT), que foi em triplicata e obtida mediana (7).

Os dados de peso e altura foram coletados segundo o proposto por Gibson (7). O peso foi obtido com balança portátil tipo plataforma, digital e eletrônica, marca Kratos-Cas (modelo Linea), com capacidade máxima de 150 quilos e sensibilidade de 50 gramas. A altura foi obtida utilizando-se estadiômetro marca Seca (modelo 206), com variação de $1 \mathrm{~mm}$ e extensão máxima de $220 \mathrm{~cm}$, afixado à parede sem rodapé.

As medidas de circunferência do braço $(\mathrm{CB})$ e da cintura (CC) foram realizadas com fita métrica flexível inextensível, com comprimento máximo de $150 \mathrm{~cm}$ e variação de $1 \mathrm{~mm}$. Para a coleta foram seguidas as recomendações de Frisancho (8) e Gibson (7), respectivamente. A DCT foi aferida utilizando-se adipômetro (marca Lange - Cambridge Scientific Industries, Inc., Cambridge, Maryland, EUA) com escala até $60 \mathrm{~mm}$ e precisão de $1 \mathrm{~mm}$.

Para a avaliação antropométrica dos adolescentes, foi utilizado o índice de massa corporal para idade (IMC/I) (9), sendo a classificação desses índices, segundo os pontos de corte, expressos em percentis, proposta pelo Sistema de Gestão Federal/Estadual da Vigilância Alimentar e Nutricional - SISVAN (10). Quanto a $\mathrm{CB}$, circunferência muscular do braço $(\mathrm{CMB})$, área do braço $(A B)$, área muscular do braço $(A M B)$, área de gordura do braço (AGB) e DCT, utilizaram-se os percentis propostos por Frisancho (8). Em relação à CC, empregaram-se os pontos de corte propostos por Taylor e cols. (11). 


\section{Avaliação do controle metabólico e insulinoterapia}

A hemoglobina glicada (\%) (AlC) foi obtida por meio de exames laboratoriais de rotina dos ambulatórios e apresentados na consulta, e os dados referentes à insulinoterapia, por meio de entrevista e consulta aos prontuários. Esses dados foram registrados em questionário próprio. Os dados para $\mathrm{AlC}$ (\%), por terem sido realizados em laboratórios diferentes, com metodologias distintas (Cromatografia Iônica, Trivelli Modificado e Cromatografia Líquida de Alta Eficiência), foram categorizados em "controle adequado" para valores até o limite máximo de referência do método, e "controle inadequado" para resultados superiores.

\section{Avaliação do consumo alimentar}

Foram aplicados quatro recordatórios de 24 horas (R24h), em intervalos de 30 dias. O primeiro foi aplicado no dia da consulta e o restante por telefone, em diferentes dias da semana, sendo pelo menos um no sábado ou domingo. $\mathrm{O}$ cálculo do consumo médio de energia, carboidrato, proteína, lipídeos e fibra foi realizado em planilha do Windows Microsoft Excel elaborada para esse fim, cujos dados de composição química de alimentos foram compilados de tabelas brasileiras (12-14), como também de rótulos de alimentos industrializados e alimentos formulados.

\section{Avaliação do nível de atividade física e necessidade estimada de energia}

A prática de atividades diárias foi registrada em formulário dividido em 48 períodos de 30 minutos. Segundo o Institute of Medicine (15), cada atividade tem um coeficiente de atividade física, de acordo com a idade e o gênero. Para determinar o nível de atividade física (NAF), foi realizada a soma dos coeficientes de atividade física e posterior divisão pelo total de períodos.

A classificação do NAF foi determinada por meio dos intervalos, sendo sedentário quando maior ou igual a 1,0 e menor que 1,4 ; leve quando maior ou igual a 1,4 e menor que 1,6 ; moderado quando maior ou igual a 1,6 e menor que 1,9 ; e intenso quando maior ou igual a 1,9 e menor que 2,5 (15).

Para o cálculo da necessidade estimada de energia (NEE), foram utilizadas as equações para adolescentes, por gênero (15).

\section{Análise estatística}

Os dados foram armazenados em planilha do Windows Microsoft Excel e transcritos para o programa Statisti- cal Package for the Social Sciences (SPSS) versão 18.0. Empregou-se estatística descritiva em que foram obtidos frequências, médias, mediana, desvio-padrão e valor mínimo e máximo. As variáveis contínuas foram testadas quanto à normalidade pelo teste de Kolmogorov-Smirnov. Para comparar os grupos, foi utilizado teste $t$ de Student, para variáveis com distribuição normal, e Mann-Whitney para variáveis sem distribuição gaussiana. Para as variáveis categóricas, aplicou-se o teste exato de qui-quadrado e teste exato de Fisher. O limiar de significância estatística foi estabelecido em $5 \%(\mathrm{p}<0,05)$.

\section{Considerações éticas}

O estudo foi aprovado pelo Comitê de Ética em Pesquisa Médica Humana e Animal do HC ${\text { Protocolo }{ }^{\circ}}^{\circ}$ 042/07) e do HGG (Protocolo ${ }^{\circ} 383 / 08$ ). Todos os pacientes e responsáveis foram esclarecidos sobre os objetivos e procedimentos da pesquisa e, após concordarem em participar voluntariamente, assinaram o Termo de Consentimento Livre e Esclarecido conforme a Resolução 196/96 do Conselho Nacional de Saúde (16).

\section{RESULTADOS}

Do total de 84 adolescentes elegíveis para o estudo segundo os critérios de inclusão, exclusão e consentimento em participar do estudo, $71(84,52 \%)$ tiveram todos os dados completos. Não foi encontrada diferença significante entre os adolescentes que permaneceram no estudo e os que não completaram todos os inquéritos quanto às características socioeconômicas e demográficas.

Na tabela 1, são apresentados os resultados dos fatores socioeconômicos e demográficos, distribuídos segundo adequação do controle glicêmico. Observou-se que, do total do grupo, somente 13 pacientes $(18,3 \%)$ foram classificados com controle glicêmico adequado. Quanto à frequência dos gêneros, não foi encontrada diferença significante entre os grupos de controle glicêmico adequado e inadequado. Também não houve diferença significante entre as médias de idade do grupo adequado e do inadequado.

Os adolescentes apresentaram principalmente ensino fundamental incompleto $(59,2 \%)$ e, como ocupação, $55(77,5 \%)$ pacientes somente estudavam. Essas características, quando avaliadas quanto ao controle glicêmico, não apresentaram significância estatística (Tabela 1). 
Tabela 1. Distribuição das prevalências de adequação da hemoglobina glicada segundo os fatores socioeconômicos e demográficos de adolescentes portadores de diabetes tipo 1. Goiânia, G0. 2010

\begin{tabular}{|c|c|c|c|c|}
\hline \multirow{2}{*}{ Características } & \multicolumn{2}{|c|}{ A1C } & \multirow{2}{*}{ Total (\%) } & \multirow{2}{*}{$\mathbf{P}^{*}$} \\
\hline & Adequado & Inadequado & & \\
\hline \multicolumn{5}{|l|}{ Gênero } \\
\hline Feminino & $8(61,5 \%)$ & $33(59,9 \%)$ & $41(57,7)$ & \multirow[t]{2}{*}{$1,000^{\dagger}$} \\
\hline Masculino & $5(35,8 \%)$ & $25(43,1 \%)$ & $30(42,3)$ & \\
\hline Idade ${ }^{\S}$ & $\begin{array}{c}12,92 \pm 2,47 \\
(10-18)\end{array}$ & $\begin{array}{c}14,38 \pm 2,43 \\
(10-18)\end{array}$ & & $0,056^{\dagger+}$ \\
\hline \multicolumn{5}{|l|}{ Escolaridade do paciente } \\
\hline Fundamental incompleto & $10(76,9 \%)$ & $32(55,2 \%)$ & $42(59,2)$ & \multirow[t]{2}{*}{$0,215^{\dagger}$} \\
\hline Fundamental completo & $3(10,3 \%)$ & $26(89,7 \%)$ & $29(40,8)$ & \\
\hline \multicolumn{5}{|l|}{ Ocupação do paciente } \\
\hline Estuda & $12(21,8 \%)$ & $43(78,2 \%)$ & $55(77,5)$ & \multirow{2}{*}{$0,272^{\mp}$} \\
\hline Estuda e/ou trabalha & $1(6,3 \%)$ & $15(93,7 \%)$ & $16(22,5)$ & \\
\hline \multicolumn{5}{|l|}{ Parentesco do cuidador } \\
\hline Mãe & $12(18,5 \%)$ & $53(81,5 \%)$ & $65(91,5)$ & \multirow[t]{2}{*}{$1,000^{\mp}$} \\
\hline Pai/avós/irmão & $1(16,7 \%)$ & $5(83,3 \%)$ & $6(8,5)$ & \\
\hline Anos de estudo do cuidador ${ }^{\S}$ & $15(7-17)$ & $7(0-17)$ & & $0,015^{\mp F}$ \\
\hline \multicolumn{5}{|l|}{ Renda per capita (SM) } \\
\hline $0,0 \vdash 0,5$ & $9(22,0 \%)$ & $32(78,0 \%)$ & $41(57,7)$ & \multirow{3}{*}{$0,648^{\dagger}$} \\
\hline $0,5 \vdash 1,0$ & $3(15,8 \%)$ & $16(84,2 \%)$ & $19(26,8)$ & \\
\hline $1,0 \mathrm{H} 2,0$ & $1(9,1 \%)$ & $10(90,9 \%)$ & $11(15,5)$ & \\
\hline \multicolumn{5}{|l|}{$\operatorname{CEB}^{(6)}(n=70)$} \\
\hline $\mathrm{B}$ e C & $9(17,6 \%)$ & $42(82,4 \%)$ & $51(72,9)$ & \multirow[t]{2}{*}{$1,000^{\dagger}$} \\
\hline $\mathrm{D}$ & $4(21,1 \%)$ & $15(78,9 \%)$ & $19(27,1)$ & \\
\hline \multicolumn{5}{|l|}{ Zona residencial } \\
\hline Urbana & $12(18,8 \%)$ & $52(81,3 \%)$ & $64(90,1)$ & \multirow[t]{2}{*}{$1,000^{\mp}$} \\
\hline Rural & $1(14,3 \%)$ & $6(85,7 \%)$ & $7(9,9)$ & \\
\hline \multicolumn{5}{|l|}{ Rede pública de água } \\
\hline Sim & $9(15,8 \%)$ & $48(84,2 \%)$ & $57(80,3)$ & \multirow[t]{2}{*}{$0,270^{+}$} \\
\hline Não & $4(28,6 \%)$ & $10(71,4 \%)$ & $14(19,7)$ & \\
\hline \multicolumn{5}{|l|}{ Rede de esgoto } \\
\hline Sim & $5(13,2 \%)$ & $33(86,8 \%)$ & $38(53,5)$ & \multirow[t]{2}{*}{$0,357^{\dagger}$} \\
\hline Não & $8(24,2 \%)$ & $25(75,8 \%)$ & $33(46,5)$ & \\
\hline Total & $13(18,3 \%)$ & $58(81,7 \%)$ & $71(100,0)$ & \\
\hline
\end{tabular}

* Limiar de significância $\mathrm{p}<0,05$; ${ }^{\dagger}$ Teste qui-quadrado; ${ }^{+1}$ Teste $t$ de Student; ${ }^{\top}$ Teste exato de Fisher; İ Teste de Mann-Whitney; ${ }^{\circledR}$ Média \pm desvio-padrão (mínimo-máximo); $\$ \$$ Mediana (mínimo-máximo); SM: salário-mínimo; CEB: Classificação Econômica Brasileira.

Em relação ao cuidador, quanto ao parentesco, a maioria encontrada foi de mães $(91,5 \%)$. Já quanto à escolaridade do cuidador, apresentada em mediana de anos de estudo, foi estatisticamente maior no grupo de adolescentes com controle glicêmico adequado (Tabela 1).

A renda per capita mensal predominante $(57,7 \%)$ foi de menos de meio salário-mínimo e $27,1 \%$ pertenciam à classe econômica "D". Quase a totalidade $(90,1 \%)$ habitava em área urbana, mas 19,7\% dos adolescentes não consumiam água distribuída pela rede pública, e 46,5\% não possuíam esgotamento sanitário (Tabela 1 ).

$\mathrm{Na}$ tabela 2, observa-se que os pacientes com controle glicêmico inadequado apresentaram menor idade no diagnóstico e maior tempo de duração da doença.
Tabela 2. Média e distribuição das prevalências de adequação da hemoglobina glicada segundo as características da doença e de insulinoterapia de adolescentes portadores de diabetes tipo 1. Goiânia, G0. 2010

\begin{tabular}{|c|c|c|c|c|}
\hline \multirow{2}{*}{ Características } & \multicolumn{2}{|c|}{ A1C } & \multirow{2}{*}{ Total (\%) } & \multirow{2}{*}{$\mathbf{P}^{*}$} \\
\hline & Adequado & Inadequado & & \\
\hline $\begin{array}{l}\text { Idade no diagnóstico } \\
\text { (meses)§ }\end{array}$ & $\begin{array}{c}103,38 \pm 49,37 \\
(7-180)\end{array}$ & $\begin{array}{c}92,88 \pm 49,31 \\
(12-182)\end{array}$ & & $0,490^{\dagger}$ \\
\hline $\begin{array}{l}\text { Duração da doença } \\
\text { (meses) }\end{array}$ & $\begin{array}{c}54,38 \pm 55,35 \\
\quad(5-192)\end{array}$ & $\begin{array}{c}81,24 \pm 46,97 \\
(8-180)\end{array}$ & & $0,076^{\dagger}$ \\
\hline \multicolumn{5}{|l|}{ Tipo de insulina } \\
\hline Lenta & $1(25,0 \%)$ & $3(75,0 \%)$ & $4(5,6)$ & \multirow{4}{*}{0,040} \\
\hline $\mathrm{NPH}$ e regular & $7(13,5 \%)$ & $45(86,5 \%)$ & $52(73,2)$ & \\
\hline Outros & $3(23,1 \%)$ & $10(76,9 \%)$ & $13(18,3)$ & \\
\hline Nenhuma & $2(100,0 \%)$ & $0(0,0 \%)$ & $2(2,8)$ & \\
\hline Dose insulina $(\mathrm{U} / \mathrm{kg} / \mathrm{dia})^{\S}$ & $\begin{array}{c}0,64 \pm 0,41 \\
(0,0-1,19)\end{array}$ & $\begin{array}{l}0,85 \pm 0,23 \\
(0,40-1,39)\end{array}$ & & $0,010^{\dagger}$ \\
\hline$\overline{\text { Total }}$ & $13(18,3)$ & $58(81,7)$ & 71 & \\
\hline
\end{tabular}

* Limiar de significância $\mathrm{p}<0,05$; ${ }^{\S}$ Média \pm desvio-padrão (mínimo-máximo); ${ }^{\dagger}$ Teste $t$ de Student; ' Teste exato de Fisher.

Entretanto, essas variáveis não influenciaram significativamente no controle glicêmico.

Em relação à insulinoterapia, 91,5\% apresentaram esquema de múltiplas doses, principalmente de Neutral Protamine Hagedorn (NPH) e insulina regular $(73,2 \%)$ $\mathrm{e}$, quanto à dose de insulina, os adolescentes com controle glicêmico adequado usavam quantidade $(0,64 \mathrm{U} /$ $\mathrm{kg} /$ dia $\pm 0,4 \mathrm{l} ; \mathrm{p}=0,0 \mathrm{l})$ significativamente menor do que aqueles com controle glicêmico inadequado ( $\mathrm{Ta}-$ bela 2).

Foi encontrada prevalência de excesso de peso de $14,1 \%$ quanto ao IMC/idade. Desses pacientes, 90,0\% apresentaram controle glicêmico inadequado. Em relação à dobra cutânea triciptal, a prevalência de excesso de peso foi de $25,4 \%$, e de $16,9 \%$ para a área de gordura do braço. Para a circunferência da cintura, a prevalência foi de $15,5 \%$ de adolescentes acima do percentil desejável (percentil 80). As variáveis antropométricas não influenciaram significativamente no controle glicêmico (Tabela 3).

Os resultados relativos ao consumo alimentar estão apresentados na tabela 4. Os grupos de adolescentes com controle glicêmico adequado e inadequado não apresentaram diferença significante para a NEE. Já o consumo em valor energético total (VET) foi significativamente menor para aqueles com controle glicêmico inadequado. $\mathrm{O}$ consumo de carboidratos e fibras foi significativamente maior entre os adolescentes com controle glicêmico adequado. Para os demais macronutrientes e respectivas porcentagens em relação ao valor calórico total, não foram encontradas diferenças significantes entre os grupos de adequação glicêmica. 
Em relação ao coeficiente de atividade física, não houve diferença entre os grupos. Quanto à sua classificação, observou-se que 34 adolescentes $(47,9 \%)$ eram sedentários e somente nove $(12,7 \%)$ realizavam atividade moderada ou intensa. Quando comparados quanto à adequação glicêmica, não foram identificadas diferenças significantes (Tabela 4).

Tabela 3. Distribuição das prevalências de adequação da hemoglobina glicada segundo características antropométricas de adolescentes portadores de diabetes tipo 1. Goiânia, G0. 2010

\begin{tabular}{|c|c|c|c|c|c|}
\hline \multirow{2}{*}{\multicolumn{2}{|c|}{ Características }} & \multicolumn{2}{|c|}{ A1C } & \multirow{3}{*}{$\begin{array}{c}\text { Total (\%) } \\
61(85,9)\end{array}$} & \multirow{2}{*}{$P^{*}$} \\
\hline & & \multirow{3}{*}{$\begin{array}{c}\text { Adequado } \\
12(19,7 \%) \\
1(10,0 \%)\end{array}$} & \multirow{2}{*}{$\begin{array}{c}\text { Inadequado } \\
49(80,3 \%)\end{array}$} & & \\
\hline$I M C^{(10)}$ & $<$ percentil 85 & & & & \multirow{2}{*}{$0,676^{\mp}$} \\
\hline & $\geq$ percentil 85 & & $9(90,0 \%)$ & $10(14,1)$ & \\
\hline \multirow[t]{2}{*}{$\mathrm{DCT} T^{(8)}$} & $<$ percentil 90 & $10(18,9 \%)$ & $43(81,1 \%)$ & $53(74,6)$ & \multirow{2}{*}{$1,000^{\mp}$} \\
\hline & $\geq$ percentil 90 & $3(16,7 \%)$ & $15(83,3 \%)$ & $18(25,4)$ & \\
\hline \multirow[t]{2}{*}{$\mathrm{CB}^{(8)}$} & $<$ percentil 90 & $13(18,8 \%)$ & $56(81,2 \%)$ & $69(97,2)$ & \multirow{2}{*}{$1,000^{\mp}$} \\
\hline & $\geq$ percentil 90 & $0(0,0 \%)$ & $2(100,0 \%)$ & $2(2,8)$ & \\
\hline \multirow[t]{2}{*}{$\mathrm{AMB}^{(8)}$} & $<$ percentil 90 & $4(11,4 \%)$ & $31(88,6 \%)$ & $35(49,3)$ & \multirow{2}{*}{$0,220^{\dagger}$} \\
\hline & $\geq$ percentil 90 & $9(25,0 \%)$ & $27(75,0 \%)$ & $36(50,7)$ & \\
\hline \multirow[t]{2}{*}{$A G B^{(8)}$} & $<$ percentil 90 & $11(18,6 \%)$ & $48(81,4 \%)$ & $59(83,1)$ & \multirow{2}{*}{$1,000^{\dagger}$} \\
\hline & $\geq$ percentil 90 & $2(16,7 \%)$ & $10(83,3 \%)$ & $12(16,9)$ & \\
\hline \multirow[t]{2}{*}{$\mathrm{CC}^{(11)}$} & $<$ percentil 80 & $12(20,0 \%)$ & $48(80,0 \%)$ & $60(84,5)$ & \multirow{2}{*}{$0,676^{\mp}$} \\
\hline & $\geq$ percentil 80 & $1(9,1 \%)$ & $10(90,9 \%)$ & $11(15,5)$ & \\
\hline \multicolumn{2}{|l|}{ Total } & $13(18,3 \%)$ & $58(81,7 \%)$ & 71 & \\
\hline
\end{tabular}

* Limiar de significância $p<0,05 ;{ }^{\dagger}$ Teste qui-quadrado; ${ }^{\mp}$ Teste exato de Fisher. IMC: índice de massa corporal; DCT: dobra cutânea triciptal; CB: circunferência do braço; AMB: área muscular do braço; AGB: área de gordura do braço; CC: circunferência da cintura.

\section{DISCUSSÃO}

As recomendações terapêuticas para o controle do DMl em adolescentes se baseiam na reposição insulínica, adequação alimentar e prática de atividade física, para prevenção de complicações agudas e crônicas e promoção de crescimento e desenvolvimento. No planejamento e aplicação das recomendações, as características dos pacientes devem ser conhecidas para maior precisão das ações e controle (3).

A maior proporção de adolescentes com controle glicêmico inadequado encontrada no presente estudo pode indicar não adesão ao tratamento, influência de fatores ambientais e, consequentemente, maior risco de desenvolver complicações crônicas e menor qualidade de vida. Jose e cols. (17), que encontraram resultados próximos aos deste estudo, ressaltaram a dificuldade em manter níveis mais baixos de AlC, mesmo em centros de referência. Outros estudos nacionais anteriores também demonstraram valores de AlC superiores a $8 \%$ $(18,19)$.

No grupo estudado, as características de gênero e idade foram similares às apresentadas por outros autores (17-19). A escolaridade foi compatível com a média de anos de estudo encontrada para indivíduos com 14 anos de idade na região Centro-Oeste. Entretanto, a expectativa dos 15 aos 17 anos é de cursar o ensino médio (20), cuja frequência líquida (nível compatível com a idade) é um desafio no Brasil, e que foi demonstrada

Tabela 4. Média, mediana e distribuição das prevalências de adequação da hemoglobina glicada segundo os fatores de consumo alimentar e atividade física de adolescentes portadores de diabetes tipo 1. Goiânia, G0. 2010

\begin{tabular}{|c|c|c|c|c|}
\hline \multirow{2}{*}{ Características } & \multicolumn{2}{|c|}{ A1C } & \multirow{2}{*}{ Total (\%) } & \multirow{2}{*}{$\mathbf{P}^{\star}$} \\
\hline & Adequado & Inadequado & & \\
\hline Necessidade estimada de energia $(\mathrm{kcal})^{\S \S}$ & $1929(1334-2858)$ & $1875(1371-3823)$ & & $0,688^{\ddagger}$ \\
\hline Consumo energético total (kcal)\$§ & $2117(1505-3375)$ & $1710(1006-4007)$ & & $0,025^{\ddagger}$ \\
\hline Consumo total de carboidratos $(\mathrm{g})^{\S}$ & $231 \pm 67(137-356)$ & $192 \pm 63(92-373)$ & & $0,027^{\dagger+}$ \\
\hline Consumo total de proteína $(\mathrm{g})^{\S}$ & $104 \pm 36(50-191)$ & $89 \pm 31(43-225)$ & & $0,144^{\dagger+}$ \\
\hline Consumo total de lipídeos $(\mathrm{g})^{\S}$ & $85 \pm 27(48-152)$ & $78 \pm 30(31-180)$ & & $0,446^{\dagger+}$ \\
\hline Consumo total de fibras $(\mathrm{g})^{\S}$ & $41 \pm 19(13-79)$ & $32 \pm 12(12-67)$ & & $0,032^{\dagger+}$ \\
\hline Kcal de carboidrato (\%)§ & $45 \pm 8(33-59)$ & $42 \pm 7(31-63)$ & & $0,263^{\dagger t}$ \\
\hline Kcal de proteína (\%) $)^{\S}$ & $19 \pm 4(13-27)$ & $20 \pm 4(12-32)$ & & $0,763^{\dagger+}$ \\
\hline Kcal de lipídeo $(\%)^{\S}$ & $36 \pm 7(21-51)$ & $38 \pm 7(20-52)$ & & $0,337^{\dagger+}$ \\
\hline Coeficiente de atividade física ${ }^{\S}$ & $1,37 \pm 0,16(1,10-2,04)$ & $1,46 \pm 0,25(1,19-2,53)$ & & $0,214^{+t}$ \\
\hline Classificação da atividade física & $7(21 \%)$ & $27(79 \%)$ & $34(48)$ & $0,959^{\ddagger}$ \\
\hline Sedentária & $5(18 \%)$ & $23(82 \%)$ & $28(39)$ & \\
\hline Leve & $1(17 \%)$ & $5(83 \%)$ & $6(9)$ & \\
\hline Moderada & $0(0 \%)$ & $3(100 \%)$ & $3(4)$ & \\
\hline \multicolumn{5}{|l|}{ Intensa } \\
\hline Total & $13(18 \%)$ & $58(82 \%)$ & 71 & \\
\hline
\end{tabular}

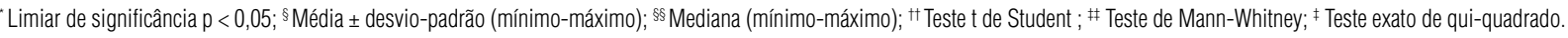


neste estudo, em que $22,5 \%$ dos adolescentes já trabalhavam para complementar a renda familiar.

A escolaridade do cuidador pode ser um indicador importante no controle glicêmico. O cuidador, principalmente representado pela mãe, assim como o demonstrado por Góes e cols. (21), apresentou mediana de anos de estudo referente ao ensino fundamental incompleto no grupo de glicemia inadequada. Paulino e cols. (19) identificaram que $69,5 \%$ das mães dos diabéticos tinham somente até 8 anos de estudo. Essa vulnerabilidade social pode dificultar a adesão ao tratamento, pela menor compreensão dos procedimentos, como até mesmo da complexidade da doença $(19,21)$.

Apesar de a renda mensal per capita não ter influenciado significativamente sobre o controle glicêmico, a prevalência de $78 \%$ dos pacientes com renda inferior a meio salário-mínimo, apresentando inadequação metabólica, reforça esse contexto. Paulino e cols. (19) também encontraram $67,8 \%$ dos diabéticos tipo 1 com renda inferior a um salário-mínimo. O Banco Mundial determina como linha de pobreza internacional o valor de 1,25 dólar por dia. No Brasil, em função de sua renda média, a linha de pobreza é definida em menos de dois dólares por dia. Em termos de salário-mínimo, são considerados indigentes, que vivem em extrema pobreza, aqueles com renda per capita menor de um quarto do salário-mínimo, e pobres, aqueles com até meio salário-mínimo (20). Portanto, a maioria dos adolescentes do estudo é classificada como pobre ou indigente.

Em estudo conduzido por Walsh e cols. (22), observou-se que baixas condições econômicas estão relacionadas à maior prevalência de complicações decorrentes do DMl. Os autores explicam que o alto custo do tratamento pode impedir que aqueles com menor renda consigam seguir as recomendações para o cuidado do diabetes, como, por exemplo, o acesso a alimentos saudáveis e atividade física. Constataram ainda que investimentos e gastos per capita com cuidado em saúde têm correlação inversa com níveis de AlC e complicações. No Estado de Goiás, Brasil, onde foi realizado o estudo, segundo a Pesquisa de Orçamento Familiar 2002-2003 (23), a proporção da renda familiar utilizada com saúde, para aqueles com renda até 400 reais, é de $12,45 \%$, e com medicamentos é de $8,78 \%$.

Hassan e cols. (24) relataram que o grupo com menor nível socioeconômico apresentou pior controle metabólico devido à disponibilidade do cuidado, assim como menor entendimento sobre a doença. Esses fatores estão diretamente relacionados à qualidade de vida desses adolescentes. Araujo e cols. (25), em estudo nacional, observaram que a baixa renda familiar influenciou negativamente sobre a qualidade de vida, com tendência a índice ruim quando inferior a dois salários-mínimos. No presente estudo, observou-se baixo nível socioeconômico e pobre controle metabólico. Atenção deve ser dada a esses fatores para adotar medidas de cuidado e prevenção.

A idade no diagnóstico e a duração da doença descritas no estudo foram semelhantes às encontradas na literatura. O maior tempo de duração da doença no grupo com controle glicêmico ruim foi observado por Jose e cols. (17), mas, também, sem influenciar significativamente nos níveis de AlC. No entanto, deve-se observar que o tempo de duração da doença, pelo longo período de vida em condições de desequilíbrio metabólico, pode levar a surgimento precoce e agravamento das complicações (4).

Ainda não foi estabelecida relação entre idade no diagnóstico e controle glicêmico. Contudo, é conhecido que a incidência de DMl vem aumentando principalmente em faixas etárias menores, em todo o mundo (26). No estudo foi observado que os adolescentes foram diagnosticados com média de idade inferior aos 10 anos de idade.

O tratamento intensivo com múltiplas doses de insulina é reconhecido como eficaz em diminuir a frequência de complicações crônicas. Nesse esquema, utilizam-se duas ou mais doses de NPH com três doses de insulina regular (3). No presente estudo, a maior parte dos adolescentes praticava esse esquema. Segundo Maniatis e cols. (27), apesar de essa terapia reduzir os níveis de $\mathrm{AlC}$, o bom controle glicêmico não tem sido alcançado. Já o uso da terapia de infusão subcutânea, ou bomba de insulina, tem proporcionado melhora significativa nos níveis de AlC, com diminuição de eventos de hipoglicemia. Mesmo sabendo-se que os análogos de insulina conseguem melhores respostas por serem mais fisiológicos e que a bomba de insulina é um padrão-ouro no tratamento intensivo (3), são necessários maiores recursos financeiros, o que impossibilita seu acesso a essa população.

A determinação da dose de insulina é um desafio. Geralmente após um ano de diagnóstico o paciente necessita de $1 \mathrm{U} / \mathrm{kg} /$ dia (3). Na adolescência pode haver uma maior necessidade devido à ação de hormônios, cuja média pode variar $31 \%$ nesse período. Essa necessidade é diferente entre os gêneros e no esquema intensivo a dose de insulina é aumentada (28). No estudo, 
o grupo com maior dose de insulina apresentou pior controle glicêmico. Jose e cols. (17) demonstraram que doses maiores de $0,8 \mathrm{U} / \mathrm{kg} /$ dia e duração da doença por mais de 5 anos foram associados a controle ruim do DMl.

Sugere-se que o tratamento prolongado em pacientes com maior tempo de diagnóstico e a busca por um bom controle glicêmico podem levar à utilização de doses suprafisiológicas de insulina e o desenvolvimento de resistência à insulina secundária. Os estudos, apesar de apresentarem resultados heterogêneos, admitem haver uma conjunção entre maior nível de hormônio, diminuição da sensibilidade à insulina, valores elevados de AlC, maior necessidade de insulina e aumento do peso corporal $(3,17,28)$.

A frequência de excesso de peso observada no estudo foi próxima à descrita por Liberatore Junior e cols. (18), que observaram prevalência de $16 \%$ de excesso de peso nos diabéticos tipo 1 . Outro estudo nacional, anterior, demonstrou tendência de excesso de peso maior entre as meninas, mas sem diferença entre os grupos de adolescentes não diabéticos (19).

O SEARCH (29), estudo norte-americano multicêntrico de base populacional, teve como objetivo identificar a prevalência de diabetes em jovens menores de 20 anos a partir do ano de 2001 . Os pesquisadores observaram que, entre os adolescentes com DMl, 34\% apresentaram sobrepeso e obesidade, o que foi comparável aos jovens não diabéticos $(33 \%)$. Em estudos na Suécia com meninas diabéticas tipo l (30) e Espanha (31), também não foram encontradas diferenças entre a prevalência de excesso de peso de diabéticos tipo $1 \mathrm{e}$ não diabéticos.

Percebe-se, então, elevada prevalência de sobrepeso e obesidade entre os diabéticos tipo 1, mas que parece acompanhar tendência de aumento ponderal entre os adolescentes sem a doença. Em estudo do estado nutricional da população brasileira de adolescentes de 20022003, a frequência de excesso de peso foi de $16,7 \%$. Verificou-se aumento, principalmente entre os meninos, desde o primeiro estudo há 14 anos, que foi de $3,8 \%$ para $18 \%$ (23). Na região Centro-Oeste, Menezes e cols. (32) descreveram prevalência de excesso de peso de $8,26 \%$, menor do que a encontrada no estudo, mas ambas elevadas.

As medidas de dobras cutâneas são consideradas por alguns pesquisadores como melhores indicadores da quantidade de gordura corporal do que o IMC (33). Paulino e cols. (19), que encontraram escore de área de gordura braquial mais elevada entre os adolescentes diabéticos tipo 1 , justificaram que o perímetro braquial e a DCT podem ter sido superestimados nos pacientes diabéticos em decorrência da lipodistrofia, comumente presente nos diabéticos nos locais onde repetidamente são aplicadas a insulina. Foi observada presença de lipo-hipertrofia nos pacientes do estudo, cuja frequência de excesso de peso pela DCT foi superior a descrita por Dunquia e cols. (33) para adolescentes sem a doença (20\% para os meninos e $14 \%$ para as meninas). Sugere-se, portanto, cautela ao utilizar os resultados dessa medida antropométrica para diabéticos em uso de insulina.

A prevalência de $15,5 \%$ de pacientes com circunferência da cintura acima do desejável associada ao excesso de peso confirma risco cardiovascular nos diabéticos, cuja doença, isolada, já apresenta esse risco aumentado. Essa frequência elevada, também presente em adolescentes sem a doença, foi demonstrada que aumenta a chance de presença de outros fatores de risco como hipertensão, dislipidemia e resistência à insulina (34). Um estudo com jovens diabéticos encontrou que $22 \%$ apresentavam história familiar de doença cardiovascular (DCV), que esses jovens tinham maior IMC e, ainda, a lipoproteína (a) esteve associada com risco de DCV precoce $(35)$.

$\mathrm{O}$ excesso de peso em adolescentes com DMl pode ser explicado pela hiperinsulinização, maior flexibilidade do consumo alimentar, mudança no gasto energético e valores elevados de hormônio do crescimento. Mesmo que no estudo não tenha sido demonstrada influência do estado nutricional sobre o controle glicêmico, a presença de sobrepeso ou obesidade nessa população deve ser considerada. Além do risco cardiovascular, o excesso de peso e a resistência à insulina podem alterar as manifestações clínicas e condutas terapêuticas do DMI (29).

Como o DMl ocorre principalmente em crianças e adolescentes e por ser uma patologia diretamente relacionada ao metabolismo de carboidratos, proteínas e gorduras, o plano nutricional associado à atividade física e à insulinoterapia é primordial no tratamento desses pacientes $(3,36)$. Ao se avaliar o consumo energético, observou-se que esse foi menor no grupo de controle glicêmico insatisfatório. Sugere-se que na avaliação de consumo alimentar em adolescentes, além da ocorrência de variabilidade intrapessoal, pacientes com excesso de peso tendem a subestimar o consumo energético, assim como omitir a ingestão de alimentos que tenham algum significado negativo como doces ou alimentos com alto teor de gordura (30). Mayer-Davis e cols. (37) 
descreveram consumo energético médio de adolescentes diabéticos tipo 1 divididos por idade, de 10 a 14 e maiores de 15 anos, de 1925,0 kcal e 2056,2 kcal, respectivamente. Esses valores foram superiores aos do estudo.

Quanto aos macronutrientes, Mayer-Davis e cols. (37) apresentaram valores semelhantes aos observados neste trabalho. Overby e cols. (38) demonstraram que o consumo de carboidrato foi superior, e de proteína e lipídios foi inferior, quando analisados por porcentagem de contribuição energética. Os autores também descreveram que o maior consumo de fibra apresentou associação com melhor controle glicêmico. No presente estudo, o consumo de fibra foi maior no grupo de controle glicêmico adequado. Estudos nacionais com adolescentes sem diabetes, ao contrário dos pacientes do estudo, apresentaram consumo maior de carboidrato e menor de proteína e lipídios (39).

Como não existem recomendações específicas para crianças e adolescentes portadores de DMl, as diretrizes da American Diabetes Association são as mesmas aplicadas para indivíduos saudáveis. Para carboidrato recomenda-se a distribuição energética de $45 \%$ a $65 \%$, para proteína, de $10 \%$ a $30 \%$ de energia, e para lipídios, de $25 \%$ a $35 \%$. A recomendação para fibra é de 20 a $35 \mathrm{~g} /$ dia $(3,36)$. Foi observado desequilíbrio na ingestão de nutrientes, sendo o consumo hipoglicídico e hiperlipídico. O consumo de proteína e fibras esteve adequado.

Uma avaliação por período de tempo maior e com instrumentos que possam identificar as principais fontes alimentares, como um questionário de frequência alimentar validado, poderia auxiliar nas orientações nutricionais para esses pacientes, cujo controle metabólico é na maioria ruim e a frequência de excesso de peso é elevada. A educação nutricional é uma opção válida e viável para os pacientes, cuidadores e toda a equipe de saúde, para que tabus e antigos conceitos de restrições alimentares sejam corrigidos, e a alimentação saudável, que fornece todos os nutrientes necessários, seja contemplada (3).

Quanto à atividade física, foi elevada a frequência de adolescentes classificados como sedentários ou somente executando atividade leve, principalmente no grupo com controle glicêmico inadequado. Segundo Elkins e cols. (40), a principal fonte de atividade física dos adolescentes está relacionada a atividades na escola, até mesmo do trajeto se é feito a pé ou de bicicleta. Em momentos fora da escola, em casa, costumam assistir à televisão, jogar jogos eletrônicos ou permanecer no computador. Os autores sugerem que atividades extracurriculares poderiam aumentar o nível de atividade física e gasto energético.

Em um contexto de baixo nível socioeconômico, educacional, acesso à saúde e aumento de prevalência de fatores de risco, a perspectiva de insegurança e de baixa qualidade de vida pode ser uma constante no cotidiano desses pacientes e seus familiares. A formação de grupos de apoio, constituídos por profissionais multidisciplinares, que auxiliem esses pacientes com atividades assistenciais e educativas, pode ser uma alternativa para que visualizem um futuro melhor e mais duradouro $(25,29)$.

O estudo apresentou limitações que podem impedir conclusões mais precisas ou que sejam extrapoladas para outras populações, como o tamanho da amostra e a determinação de AlC por métodos e laboratórios diferentes. O ideal é que sejam formados grupos multicêntricos e realizados estudos longitudinais prospectivos, com metodologias padronizadas, para se avaliar causas e efeito. Contudo, os relevantes resultados encontrados podem ser úteis ao planejamento de ações e estudos que promovam a melhora da qualidade de vida dos adolescentes portadores de DMl.

Logo, os achados do presente estudo permitem concluir que é elevada a proporção de adolescentes diabéticos tipo 1 com inadequação do controle glicêmico e alimentação desequilibrada, e que fatores como menor escolaridade do cuidador, tipo e dose de insulina influenciaram para o controle glicêmico ruim desses pacientes.

Declaração: os autores declaram não haver conflitos de interesse científico neste estudo.

\section{REFERÊNCIAS}

1. Georg AE, Duncan BB, Toscano CM, Schmidt MI, Mengue S, Duarte $C$, et al. Análise econômica do programa para rastreamento do diabetes mellitus no Brasil. Rev Saúde Pública. 2005;29(3):452-60.

2. Franco DR. Fundamentos do diabetes tipo 1: etiologia, diagnóstico e insulinoterapia. Terapêutica em diabetes. 2005;10(32):1-5.

3. Sociedade Brasileira de Diabetes. Diretrizes da Sociedade Brasileira de Diabetes. Tratamento e acompanhamento do diabetes mellitus. Aspectos clínicos e laboratoriais da hemoglobina glicada. São Paulo, SP; 2007.

4. Walsh MG, Zgibor J, Borch-Johsen K, Orchard TJ. A multinational assessment of complications in type 1 diabetes: the DiaMond sub study of complications (DiaComp) Level 1. Diab Vasc Dis Res. 2006;3(2):84-92.

5. Burnet DL, Cooper AJ, Drum ML, Lipton RB. Risk factor for mortality in a diverse cohort of patients with childhood-onset diabetes in Chicago. Diabetes Care. 2007;30(10):2559-63. 
6. Associação Brasileira de Empresas de Pesquisa. Critério padrão de classificação econômica Brasil/2008. Disponível em: http:// www.abep.org/novo/Content.aspx?Content ID=301. Acesso em: Set 10, 2010.

7. Gibson RS. Principles of nutritional assessment. 2nd ed. Oxford: Oxford University Press; 2005.

8. Frisancho AR. New norms of upper limb fat and muscle areas for assessment of nutritional status. Am J Clin Nutr. 1981;34:2540-5.

9. de Onis M, Onyango AW, Borghi E, Siyam A, Nishida C, Siekmann J. Development of a WHO growth reference for school-aged children and adolescents. Bull World Health Organ. 2007;85(9):660-7.

10. Ministério da Saúde. Sistema de Gestão Federal/Estadual da Vigilância Alimentar e Nutricional-SISVAN. Orientações para a coleta e análise de dados antropométricos em serviços de saúde. Norma técnica. Material preliminar. Brasil, 2008.

11. Taylor RW, Jones IE, Williams SM, Goulding A. Evaluation of waist circumference, waist-to-hip- ratio, and the conicity index as screening tools for high trunk fat mass, as measured by dual-energy X-ray absorptiometry, in children aged 3-19 y. Am J Clin Nutr. 2000;72:490-5.

12. Ministério do Planejamento e Orçamento. Fundação Instituto Brasileiro de Geografia e Estatística - IBGE. Estudo nacional da despesa familiar - Endef. Tabelas de composição de alimentos. 4.ed. Rio de Janeiro: Ibge; 1996.

13. Núcleo de Estudos e Pesquisas em Alimentação (NEPA). TACO Tabela Brasileira de Composição de Alimentos. 2.ed. Campinas: Nepa-Unicamp; 2006.

14. Pinheiro ABV, Lacerda EMA, Benzecry EH, Gomes MCS, Costa VMC. Tabela para avaliação de consumo alimentar em medidas caseiras. 4.ed. São Paulo: Atheneu; 2002.

15. Institute of Medicine. Dietary reference intakes for energy, carbohydrate, fiber, fat, fatty acids, cholesterol, protein, and aminoacids. Washington: IOM; 2005.

16. Ministério da Saúde. Conselho Nacional de Saúde. Resolução 196 de 10 de outubro de 1996. Disponível em: http://www.datasus. gov.br/conselho/resol96/RES19696.htm. Acesso em: Set 10, 2010.

17. Jose LOS, Cardoso-Dermatini AA, Liberatore Junior RDR, Paulino MFVM, Lemos-Martini SHV, Guerra-Junior G, et al. Clinical and laboratory profile of pediatric and adolescent patients with type 1 diabetes. J Pediatr (Rio J). 2009;85(6):490-4.

18. Liberatore Junior RDR, Dermatini AAC, Ono AHA, Andrade GC. Prevalência de obesidade em crianças e adolescentes com diabetes melito tipo 1. Rev Paul Pediatr. 2008;26(2):142-5.

19. Paulino MFVM, Lemos-Marini SHV, Guerra-Junior G, Minicucci WJ, Mendes CT, Morcillo AM. Crescimento e composição corporal de crianças com diabetes mellitus tipo 1. Arq Bras Endocrinol Metab. 2006;50(3):490-8.

20. Instituto Brasileiro de Geografia e Estatística. Pesquisa nacional por amostra de domicílios 2008. Brasil. Rio de Janeiro: IBGE, v. 29, 2009. Disponível em: http://www.ibge.gov.br/home/estatistica/populacao/condicaodevida/indicadoresminimos/sinteseindicsociais2009/indic_sociais2009.pdf. Acesso em: Set 10, 2010.

21. Góes APP, Vieira MRR, Liberatore-Junior RDR. Diabetes mellitus tipo 1 no contexto familiar e social. Rev Paul Pediatr. 2007;25(2):124-8.

22. Walsh MG, Zgibor J, SongerT, Borch-Johnsen K, Orchard TJ. The socioeconomic correlates of global complication prevalence in type 1 diabetes (T1D): a multinational comparison. Diabetes Res Clin Prac. 2005;70(2):143-50.

23. Instituto Brasileiro de Geografia e Estatística. Pesquisa de Orçamentos Familiares (POF) 2002-2003: antropometria e análise do estado nutricional de crianças e adolescentes no Brasil. Rio de Janeiro, 2006. Disponível em: http://www.ibge.gov.br/home/esta- tistica/populacao/condicaodevida/pof/2003medidas/pof2003medidas.pdf. Acesso em: Set 10, 2010.

24. Hassan K, Loar R, Anderson BJ, Heptulla RA. The role of socioeconomic status, depression, quality of life, and glycemic control in type 1 diabetes mellitus. J Pediatr. 2006;149(4):526-31.

25. Araujo AF, Souza MEA, Menezes CA. Qualidade de vida e aspectos socioeconômicos em diabéticos tipo 1. Arq Bras Endocrinol Metab. 2008;52(7):1124-30.

26. Boulard $S, A t L$, Llanas $P$, Barat $P$. Caractéristiques épidémiologiques des enfants de moins de 15 ans pris en charge à l'hopital dês enfants de Bordeaux de 1998 à 2002 devant la découverte d'un diabète type 1. Arch Pédiatr. 2006;14:196-201.

27. Maniatis AK, Klingensmith GJ, Slover RH, Mowry CJ, Chase P. Continuous subcutaneous insulin infusion therapy for children and adolescents: an option for routine diabetes care. Pediatrics. 2001;107(2):351-6.

28. Wiegand S, Raile K, ReinehrT, Hofer S, Näke A, Rabl W, et al. Daily insulin requirement of children and adolescents with type 1 diabetes: effect of age, gender, body mass index and mode of therapy. Eur J Endocrinol. 2008;158:543-9.

29. Liu LL, Lawrence JM, Davis C, Liese AD, Pettitt DJ, Pihoker C, et al. prevalence of overweight and obesity in youth with diabetes in USA: the SEARCH for Diabetes in Youth Study. Pediatric Diabetes. 2010;11:4-11.

30. Särnblad S, Ekelund U, Aman J. Dietary fat intake predicts 1-year change in body fat in adolescent girls with type 1 diabetes. Diabetes Care. 2006;29(6):1227-30.

31. Gámez MAV, Pérez JMM, García MJM, García RMM, Martín FA, Cano RP. Evolución de la massa ósea durante la infância y adolescência em niños com diabetes mellitus tipo 1. Medicina Clinica (Barc). 2008;130(14):526-30.

32. Menezes IHCF, Neutzling MB, Taddei JAAC. Risk factors for overweight and obesity in adolesnts of a Brazilian university: a case-control study. Nutrición Hospitalaria. 2009;24:17-24.

33. Dunquia RP, Dunmith SC, Reichert FF, Madruga SW, Duro LN, Menezes AMB, et al. Epidemiologia das pregas cutâneas triciptal e subescapular elevadas em adolescentes. Cad Saude Publica. 2008;24(1):113-21.

34. Denney-Wilson E, Hardy L, Dobbins T, Okely AD, Baur LA. Body mass index, waist circumference, and chronic disease risk factors in Australian Adolescents. Arch Pediatr Adolesc Med. 2008;162(6):566-73.

35. Glowinska G, Urban M, Koput A. Cardiovascular risk factors in children with obesity, hypertension and diabetes: lipoprotein(a) levels and body mass index correlate with family history of cardiovascular disease. Eur J Pediatr. 2002;161:511-8.

36. Lottenberg AMP. Características da dieta nas diferentes fases da evolução do diabetes melito tipo 1. Arq Bras Endocrinol Metab. 2008;52(2):250-9.

37. Mayer-Davis EJ, Nichols M, Liese AD, Bell RA, Dabelea DM, Johansen JM, et al. Dietary intake among youth with diabetes: the SEARCH for Diabetes in Youth Study. J Am Diet Assoc. 2006;106:689-97.

38. Overby NC, Margeirsdottir HD, Brunborg C, Andersen LF, Dahl-Jorgensen $\mathrm{K}$. The influence of dietary intake and meal pattern on blood glucose control in children and adolescents using intensive insulin treatment. Diabetologia. 2007;50:2044-51.

39. Toral N, Slater B, Silva MV. Consumo alimentar e excesso de peso de adolescentes de Piracicaba, São Paulo. Rev Nutr Campinas. 2007;20(5):449-59.

40. Elkins WL, Cohen DA, Koralewics LM, Taylor SN. After school activities, overweight, and obesity among inner city youth. J Adolesc. 2004;27(2):181-9. 